\title{
Stendhal et l'Héroïsme au Féminin, éd. Michel Arrous
}

\section{Annalisa Bottacin}

\section{OpenEdition}

\section{Journals}

\section{Edizione digitale}

URL: http://journals.openedition.org/studifrancesi/5371

DOI: 10.4000/studifrancesi.5371

ISSN: 2421-5856

\section{Editore}

Rosenberg \& Sellier

\section{Edizione cartacea}

Data di pubblicazione: 1 dicembre 2016

Paginazione: $541-542$

ISSN: 0039-2944

Notizia bibliografica digitale

Annalisa Bottacin, «Stendhal et l'Héroïsme au Féminin, éd. Michel Arrous », Studi Francesi [Online], 180 (LX | III) | 2016, online dal 01 janvier 2017, consultato il 18 septembre 2020. URL : http:// journals.openedition.org/studifrancesi/5371 ; DOI : https://doi.org/10.4000/studifrancesi.5371

Questo documento è stato generato automaticamente il 18 settembre 2020.

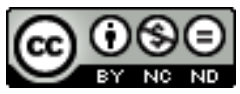

Studi Francesi è distribuita con Licenza Creative Commons Attribuzione - Non commerciale - Non opere derivate 4.0 Internazionale. 


\title{
Stendhal et l'Héroïsme au Féminin, éd. Michel Arrous
}

\author{
Annalisa Bottacin
}

\section{NOTIZIA}

Stendhal et l'Héroïsme au Féminin, actes du Colloque Paris-Sorbonne 29-30 mars 2013, études réunies et présentées par Michel Arrous, Paris, Eurédit, 2015, 240 pp.

1 La questione del «femminile» attraversa tutta la poetica stendhaliana come tematizzazione di uno spazio ideologico ed epistemologico che, oltrepassata la soglia di una condizione chiusa e limitante - come era ancora nella tradizione dell'epoca salvo alcuni esempi che non tralasciarono di far scalpore - si articola in prese di posizione anelanti al «nuovo»e al «vero». In questo corposo e interessante volume si assiste al tentativo di delineare i contorni di questa problematica, attraverso dettagliate analisi di eroine, su cui si organizza un discorso articolato per lo più basato sulla loro riconosciuta «modernità». La donna stendhaliana, è dolce ma anche audace, coraggiosa, orgogliosa, e pronta al sacrificio, eroina anelante la libertà. Come ben evidenzia il curatore Michel ARRous nell' Introduction (pp. 7-9), «[d]isciple d'Helvétius, Stendhal comprend la femme "en juge du mérite de l'homme" et considère que ce qui définit l'homme, c'est le regard de la femme: sans elle, pas de grand homme». Uno sguardo che può essere dolce, delicato, ma a volte anche terribile nella sua spietatezza, specie quando deve difendere il suo uomo o immolarsi per lui. È su questa tematica che esordisce Pierrette PAVET in un interessante studio dal titolo, Le courage des héroïnes dans le roman stendhalien (pp.11-23), in cui analizza, dalla Chartreuse a Lamiel ad alcune novelle per lo più inachevées, l'esuberanza de «l'empire des femmes» il cui maggiore merito è quello di avere «le courage d'aimer». Tre interventi hanno per soggetto Armance, primo romanzo stendhaliano, uscito nel 1827. L'accurato "Armance": éléments pour une typologie de la femme di Brahim KAROUI (pp. 25-34), il dettagliato studio di María Vicenta HERNÁNDEZ ÁLVAREZ, Les mots d"“Armance": notes pour l'analyse d'un discours au 
féminin (pp. 35-76) e l'approfondito Le «projet héroïque» d'Armance de Zohiloff di Sacha LUETHY (pp. 67-76). B. KAROUI si chiede se la protagonista del récit, Armance de Zohiloff, non riveli nei salotti della Restaurazione accanto alla passione amorosa per Octave de Malibert, quei percepibili segnali che possono considerarsi piccoli atti di eroismo. Di qui la riflessione del critico se «l'héroïsme peut [...] se vivre au féminin» (p. 25). Il secondo apporto di María Vicenta HERNÁNDEZ ÁLVAREZ puntualizza in primo luogo nel romanzo l'importanza del ruolo di Armance, la cui forza d'animo supera quella del protagonista e, con varie esemplificazioni, giunge ad avvicinarla alle eroine del passato, divisa com'è tra «l'honneur et le bonheur» (p. 53). Il «projet héroïque» di Armance è invece considerato da Sacha LUETHY «un mensonge»; in effetti, pur essendo innamorata d'Octave, la fanciulla reputa che «[d]epuis quelque temps il est question d'un mariage pour [elle]» (p. 67). Il contributo di Béatrice DIDIER (L'Héroïsme féminin à l'opéra, pp. 77-85) prende spunto dalla passione di Stendhal per il melodramma; in effetti sia l'eroismo dei personaggi che il vissuto degli stessi cantanti non sono mai indifferenti a Henri Beyle, che fa trapelare le sue opinioni non solo dai suoi scritti sulla musica, ma in special modo dai suoi reportages musicali pubblicati su varie riviste, tra cui il «Journal de Paris ", tra il 1824 e il 1827. Acutamente Béatrice Didier si chiede se questo eroismo trapeli dal genere serio o anche da quello comico: «[d]écidément l'opéra réclame de l'héroïsme à tous les niveaux: chez les personnages, chez les cantatrices, chez le critique» (p. 83). L'intervento di Max ANDRÉOLI (Héroïnes héroïques: Stendhal et Balzac, pp. 87-101), nella sua comparazione tra gli atti d'eroismo delle protagoniste di Stendhal e quelle di Balzac, si dimostra assai pertinente nell'analisi dei differenti registri dell'eroismo femminile. "La nouvelle Mina de Vanghel [...] séduit par l'envolée imaginaire, l'exaltation de la figure féminine et sa destinée tragique», scrive Suzel ESQUIER nel suo denso intervento "Mina de Vanghel": un conte qui finit mal (pp. 103-113), evidenziando quanto la tragicità del personaggio sia legata a un lirismo interiore il quale incorpora la frase musicale, specie mozartiana, «qui surgit comme un secours improvisé» (p. 109). L'articolato intervento dal titolo, Les héroïnes de Stendhal: contrepoids aux passions démocrates (pp.115-144) di Béatrice LEVET, tratta nella prima parte (pp. 115-120) l'influenza su Stendhal delle nuove idee del philosophe de l'histoire, Alexis de Toqueville e del suo De la Démocratie en Amérique, prendendone spunto per considerazioni generali sulla tendenza della società moderna verso la democrazia. L'A. sottolinea quidi che «dans l'univers stendhalien, ce sont les femmes qui maintiennent la flamme dans cette Europe révolutionnée, ce sont elles qui sont porteuses de référence, de valeurs, de principes rebelles aux critères d'efficacité, d'utilité, de rentabilité qui commandent désormais les esprits, c'est à elles que le romancier confie la tâche de prendre soin de cette noble idée de l'homme dont font litière les temps démocratiques....» (p.122). L'ennui di Lamiel o di Méthilde de la Môle, ad esempio, esprime tutte le incongruenze e i disagi de «ces mœurs nouvelles» in quanto «[d]es révolutions américaine et française est sorti un monde nouveau» (p. 125). Di estremo interesse, per una nuova visione della figura di Louise de Rênal, il saggio di Michel CROUZET (Madame de Rênal ou la tendresse héroïque, pp.145-184) che si addentra acutamente nelle pagine del Rouge con la grande maestria di chi, da tempo, studia ai più alti livelli il capolavoro stendhaliano. L'intervento è suddiviso in vari sottocapitoli, che ben evidenziano la circolarità dell'esegesi: Une Hérö̈ne paradoxale (pp. 145-53), La carte stendhalienne du Tendre (pp. 153-160), Une Grande Dame (pp. 160-163), Tendresse et Passion. Le refus de la tendresse (pp.163-168), La Tendresse contre le ressentiment (pp. 168-180). Donna dolce, di grande modestia, tratti che forse avrebbero potuto allontanarla dalla 
dimensione di eroina, Louise de Rênal è vista dal grande stendhaliano come "une étrange héroïne de la tendresse» (p.163) nella Francia moralizzante e restaurata; scevro da ogni interesse, il suo cuore è investito di un amore al di là di ogni convenzionalità che, una volta svelatosi, la erge a eroina, in una vertigine del limite che la condurrà, poco dopo l'esecuzione dell'amato Julien, alla morte. Thierry OZWALD in De "Lamiel", ou l'éternel héroïque féminin (pp.185-205) con grande acutezza analizza un personaggio che lo «intrigue fort; [Lamiel] laisse généralement perplexe la critique d'hier et d'aujourd'hui qui ne sait comment classer, aborder, envisager ce récit énigmatique» e pone vari confronti sulla lettura data all'opera dai critici. Ozwald pone la «fille du diable» di fronte ad altre eroine stendhaliane, esaltandone la grande modernità e in special modo la sua visione della libertà. Altra testimonianza attestante la grandezza di uno straordinario récit che la fine improvvisa dell'autore ha impedito di portare a termine. Di notevole rilievo lo studio di Andrée MANSAU (Héroïnes espagnolistes: Suora Scolastica et Eugenia Palafox, pp. 207-217) che analizza i possibili legami tra Suora Scolastica, una delle ultime eroine stendhaliane, e Eugenia Guzmán y Palafox, poi contessa de Montijo e Imperatrice dei Francesi, che fu legata a Stendhal da sincera amicizia, di cui resta traccia anche nella Correspondance. Conclude il bel volume la pubblicazione da parte di Barbara Innocenti delle lettere di Giulia Rinieri de' Rocchi al futuro coniuge Giulio Martini ("Je te laisse avec la plume mais pas avec le coeur...» La correspondance de Giulia Rinieri de' Rocchi avec Giulio Martini, pp. 219-238). Incontrata dopo una pena d'amore, un'attenuazione al dolore per Henri Beyle fu, «à partir de ce fatal mois de janvier 1827, une jeune Siennoise [...], sa présence apaisait déjà le tourment où le plongeait encore la seule pensée de Menti». (H. MARTINEAU, Le cœur de Stendhal, II, Paris, Albin Michel, 1953, p. 92). Come nota Innocenti, Giulia si legherà a lui di un affetto profondo al punto che, anche dopo il matrimonio con Giulio Martini, non pose fine ai loro rapporti. Nel 1896, il letterato e uomo politico Ferdinando Martini scoprì in un baule alcune carte appartenute alla zia Giulia, tra cui la lettera di Stendhal in cui la giovane veniva richiesta al tutore Daniello Berlinghieri in matrimonio, con l'esito ben noto. Queste lettere sono andate tutte perdute, e grande fu il rammarico di Luigi Foscolo Benedetto, che scrisse su Giulia pagine memorabili. Ora, Barbara Innocenti presenta una documentazione, in gran parte inedita, depositata presso l'Archivio Marchini della Bibilioteca municipale Forteguerriana di Pistoia che comprende le lettere scritte da Giulia al futuro marito, Giulio Martini, nell'aprile del 1833 (non sono espressi dalla scrivente il mese e l'anno ma solo il giorno della settimana), «avec d'autres de second plan» ad altri fruitori (p. 221), lettere che ben evidenziano la forza di carattere e la nobiltà dei sentimenti della giovane senese. Sarebbe stato a nostro avviso meno dispersivo, per una maggiore coerenza del discorso, presentare la traduzione francese delle missive in apparato o in un'unica nota al testo, comunque riconosciamo lo sforzo della curatrice nell'addentrasi in un terreno quanto mai periglioso quale è quello stendhaliano. È anche viva la speranza di nuovi ritrovamenti atti a presentare, in modo sempre più approfondito, un ritratto di colei che fu presenza determinante nell'esistenza di Henri Beyle. 